\title{
Effects of Lévy noise on the dynamics of sine-Gordon solitons in long Josephson junctions
}

\author{
Claudio Guarcello ${ }^{1,2}$, Davide Valenti ${ }^{1}$, Angelo Carollo ${ }^{1,2}$ \\ and Bernardo Spagnolo ${ }^{1,2,3}$ \\ ${ }^{1}$ Dipartimento di Fisica e Chimica, Group of Interdisciplinary Theoretical \\ Physics, Università di Palermo and CNISM, Unità di Palermo, Viale delle \\ Scienze, Edificio 18, 90128 Palermo, Italy \\ ${ }^{2}$ Radiophysics Department, Lobachevsky State University, 23 Gagarin \\ Avenue, 603950 Nizhny Novgorod, Russia \\ ${ }^{3}$ Istituto Nazionale di Fisica Nucleare, Sezione di Catania, Via S. Sofia 64, \\ I-95123 Catania, Italy \\ E-mail: claudio.guarcello@unipa.it
}

Received 22 October 2015

Accepted for publication 20 November 2015

Published 20 May 2016

Online at stacks.iop.org/JSTAT/2016/054012

doi:10.1088/1742-5468/2016/05/054012

\begin{abstract}
We numerically investigate the generation of solitons in currentbiased long Josephson junctions in relation to the superconducting lifetime and the voltage drop across the device. The dynamics of the junction is modelled with a sine-Gordon equation driven by an oscillating field and subject to an external non-Gaussian noise. A wide range of $\alpha$-stable Lévy distributions is considered as a noise source, with varying stability index $\alpha$ and asymmetry parameter $\beta$. In junctions longer than a critical length, the mean switching time (MST) from the superconductive to the resistive state assumes a value independent of the device length. Here, we demonstrate that this value is directly related to the mean density of solitons which move into or from the washboard potential minimum corresponding to the initial superconductive state. Moreover, we observe: (i) a connection between the total mean soliton density and the mean potential difference across the junction; (ii) an inverse behaviour of the mean voltage in comparison with the MST, with varying the junction length; (iii) evidence of non-monotonic behaviours, such as stochastic resonant activation and noise-enhanced stability, of the MST versus the driving frequency and noise intensity for different values of $\alpha$ and $\beta$; (iv) finally, these
\end{abstract}


non-monotonic behaviours are found to be related to the mean density of the solitons formed along the junction.

Keywords: mesoscopic systems (theory), stochastic processes (theory), metastable states, large deviations in non-equilibrium systems

\section{Contents}

1. Introduction

2. The model

3. The Lévy statistics

4. Computational details $\quad 8$

5. Results 9

5.1. Results as a function of $L \ldots \ldots \ldots \ldots \ldots$

5.2. Results as a function of $\omega \ldots \ldots \ldots \ldots \ldots$

5.3. Results as a function of $\gamma \ldots \ldots \ldots \ldots \ldots$

$\begin{array}{ll}\text { 6. Conclusions } & 18\end{array}$

$\begin{array}{ll}\text { References } & 19\end{array}$

\section{Introduction}

The problem of detecting a sinusoidal signal corrupted by a Gaussian noise source has already been, at least in principle, completely solved [1]. However, when the amount of data to process is huge or the signal-to-noise ratio is too small, the choice of detection strategy becomes crucial. As an example, we can remember the all-sky all-frequency search for gravitational waves emitted by a pulsar [2], the search for a continuous monochromatic signal in radio astronomy [3] and the detection of terahertz radiation [4]. The use of bistable systems as non-linear devices for signal detection has been recently proposed $[5,6]$ as a way of tackling these problems. Nonlinear elements inserted in place of linear-matched filters may not enhance the overall detection performance [7], although they can greatly improve the detection strategy and/or reduce the computational and memory overheads.

On account of their peculiar properties, Josephson junctions (JJs) stand out among other nonlinear elements as well-suited candidates for signal detection [5, 8]. Notably, they are very fast elements, which can operate at frequencies as high as terahertz [9]. Moreover, the thermal noise effect on JJs can be greatly reduced, cooling them down quite close to absolute zero, up to the quantum noise limit [10]. Various proposals for the use of JJs as detectors of weak signals in the presence of noise have been put forward so 
far. Some of them make use of superconducting quantum interference devices (SQUIDs) $[11,12]$, while others focus instead on the switching from the metastable superconducting state to the resistive running state of the JJs. In the latter case, various approaches have been exploited. The statistical analysis of the switching can be used to reveal weak periodic signals embedded in a noisy environment [13-17]. The rate of switching, on the other hand, can provide information about the noise present in an input signal [18-22]. Proposals to use the statistics of the escape times for signal detection have also been put forward [13, 14, 18-22].

Experimentally, many systems exhibit non-Gaussian noise signals [23-28]. For example, an out-of-equilibrium heat reservoir can be regarded as a source of non-Gaussian noise [25-27]. An example, which can be well modelled by an $\alpha$-stable distribution, can be found in a wireless ad hoc network with a Poisson field of co-channel users [28]. In current base JJs, coupled with non-equilibrium current fluctuations, the effects of nonGaussian noise on the average escape time from the superconducting metastable state have been experimentally investigated [23, 24]. Moreover, the role of Gaussian [29-38] and non-Gaussian [18-21, 39-43] noise sources on both long and short JJs have been theoretically analysed.

In this paper, we study the escape time from a metastable state of a long JJ driven by an external oscillating force and subject to a noise signal. The main quantity of interest is the mean switching time (MST), i.e. the average time the junction needs to switch from the superconducting state to the resistive regime, calculated on a sufficiently large number of numerical realisations. The analysis is performed by varying the junction length, the frequency of the driving current and the amplitude of the noise signal. The noise is modelled by an $\alpha$-stable Lévy distribution. These statistics aim at describing real situations [44] in which the variables show abrupt jumps and very rapid variations called Lévy flights.

Lévy-type statistics were observed in various research fields, characterised by the presence of scale-invariance [45-49]. The results on the Lévy flights were recently reviewed in [50]. Moreover, the Lévy statistics allowed the good reproduction of several observed evolutions in different scientific areas [51-53], ranging from zoology [54, 55], biology [56-58], population dynamics [59-61], to atmospheric [62] and geological data [63], financial markets [64], networks [65], social systems [66], signal detection [67] and solid-state physics [68-72]. An extensive bibliography on $\alpha$-stable distributions/ processes and their applications is maintained by Nolan [73].

The dynamics of the phase difference across a long JJ is well described by the sineGordon equation, which admits very peculiar wave packet solutions called solitons $[74,75]$. They can be pictured as a $2 \pi$ twist of the order parameter $\varphi$, developing and propagating along the junction. These twists carry magnetic flux quanta, i.e. fluxons, $[76,77]$, which can be observed during the switching towards the resistive state.

This paper is organised as follows. In the next section the sine-Gordon model is presented. In section 3 the statistical properties of the Lévy noise are briefly reviewed, showing some peculiarities of different $\alpha$-stable distributions. Section 4 gives the computational details. In section 5 the theoretical results as a function of the junction length, the driving frequency and the noise intensity are shown and analysed. Finally, in section 6 the conclusions are drawn. 


\section{The model}

The behaviour of a long overlap JJ is described by a nonlinear partial differential equation for the order parameter $\varphi$, the sine-Gordon (SG) equation [78, 79]

$$
\frac{\hbar}{2 e} C \frac{\partial^{2} \varphi}{\partial \mathrm{T}^{2}}+\frac{\hbar}{2 e} \frac{1}{R_{N}} \frac{\partial \varphi}{\partial \mathrm{T}}+J_{c} \sin \varphi=\frac{\hbar c^{2}}{8 \pi e d} \frac{\partial^{2} \varphi}{\partial \mathrm{x}^{2}}+\frac{2 e}{\hbar} J .
$$

Here $\varphi$ is the phase difference between the wave functions describing the superconducting condensate in the two electrodes. Hereafter, small (capital) letters are used to indicate normalised (non-normalised) coordinates and current terms (with respect to the critical value $I_{\mathrm{c}}$ ). In our JJ model, the junction is extended along only one direction, defined as x, and can be considered 'short' along the other directions, so that a magnetic field through the junction points orthogonally to the x direction. In equation (1) $J_{c}$ is the critical current density, $\hbar$ is the Planck constant, $e$ is the value of the electron charge, $c$ is the speed of light, $R_{N}$ and $C$ are the effective normal resistance and capacitance of the junction, respectively. The magnetic penetration $d=\lambda_{\mathrm{L}}+\lambda_{\mathrm{R}}+t_{i}$ is the sum of the London depths in the left and right superconductors $\lambda_{\mathrm{L}}$ and $\lambda_{\mathrm{R}}$, respectively, and the interlayer thickness $t_{i}$. The current density $J$ includes both the bias and the fluctuating current.

The SG equation can be recast [78] in terms of the dimensionless $x=\mathrm{x} / \lambda_{\mathrm{J}}$ and $t=\mathrm{T} \omega_{\mathrm{J}}$ variables, that are the space and time coordinates normalised to the Josephson penetration depth $\lambda_{\mathrm{J}}=\sqrt{\hbar c^{2} /\left(8 \pi e d J_{c}\right)}$ and to the inverse of the characteristic frequency $\omega_{\mathrm{J}}=(2 e / \hbar) I_{\mathrm{c}} R_{N}$ of the junction, respectively. Using dimensionless variables, the SG reads

$$
\beta_{c} \varphi_{t t}(x, t)+\varphi_{t}(x, t)-\varphi_{x x}(x, t)=i_{b}(x, t)-\sin (\varphi(x, t))+i_{\mathrm{f}}(x, t) .
$$

Here a simplified notation has been used, with the subscript of $\varphi$ indicating the partial derivative in that variable. This notation will be used throughout this paper.

In equation (2), the fluctuating current density $i_{\mathrm{f}}(x, t)$ is the sum of two contributions, a Gaussian thermal noise $i_{\mathrm{T}}(x, t)$ and an external non-Gaussian noise source $i_{\mathrm{nG}}(x, t)$

$$
i_{\mathrm{f}}(x, t)=i_{\mathrm{T}}(x, t)+i_{\mathrm{nG}}(x, t) .
$$

The coefficient

$$
\beta_{c}=\omega_{\mathrm{J}} R_{N} C
$$

is the Stewart-McCumber parameter. The junctions with $\beta_{c} \ll 1$ have small capacitance and/or small resistance, and are highly damped (overdamped JJ). In contrast, the junctions with $\beta_{c} \gg 1$ have large capacitance and/or large resistance, and are weakly damped (underdamped JJ). The dimensionless terms $i_{b}(x, t)$ and $\sin (\varphi)$ of equation (2) are, respectively, the bias current and supercurrent, both normalised to the JJ critical current $I_{\mathrm{c}}$. Equation (2) is solved imposing the following boundary conditions

$$
\varphi_{x}(0, t)=\varphi_{x}(L, t)=\Gamma,
$$

where $\Gamma$ is the normalised external magnetic field. Hereinafter we impose $\Gamma=0$.

The two-dimensional time-dependent tilted potential named the washboard potential is given by 

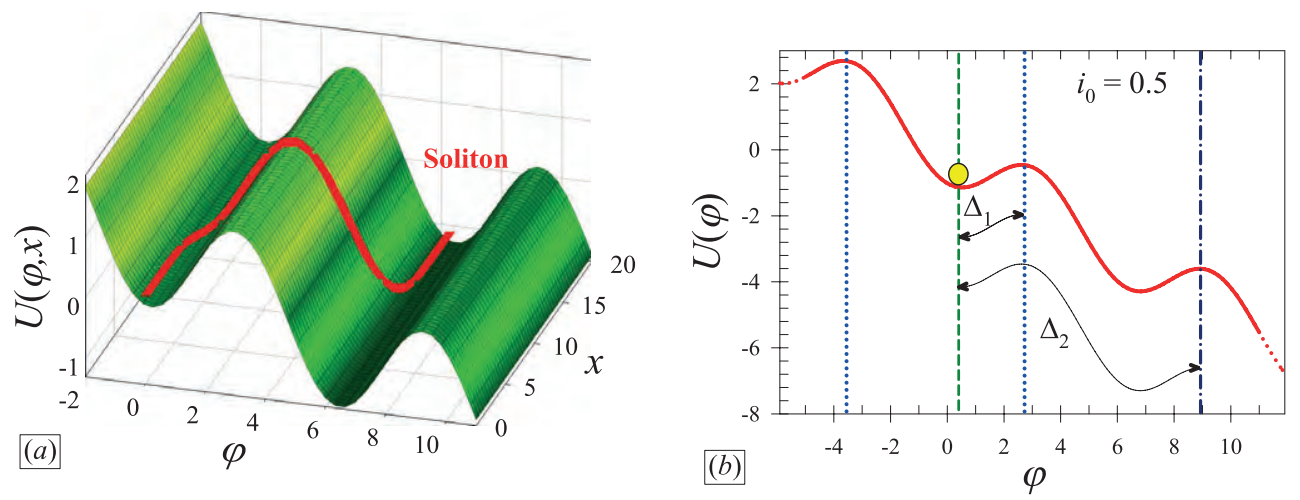

Figure 1. (a) Washboard potential in a fixed instant of its dynamics with a soliton located between two adjacent valleys. (b) Cross section of a washboard potential with slope $i_{0}=0.5$. Also shown is the initial position (the bottom of the potential well) of a cell. The blue dotted lines indicate the left and right thresholds. The picture also shows the distances along the potential profile $\Delta_{1}$ and $\Delta_{2}$ between the initial minimum and, respectively, the two successive maxima on the right.

$$
U(\varphi, x, t)=1-\cos (\varphi)-i_{b}(x, t) \varphi,
$$

and is shown in panel (a) of figure 1. In the same figure is shown a phase string along the potential profile given in equation (6). Specifically, the washboard potential is composed of a periodical sequence of peaks and valleys, with the minima and maxima satisfying the following conditions

$$
\varphi_{\min }=\arcsin \left(i_{b}(x, t)\right)+2 n \pi \quad \varphi_{\max }=\left(\pi-\arcsin \left(i_{b}(x, t)\right)\right)+2 n \pi
$$

with $n=0, \pm 1, \pm 2, \ldots$

The bias current is given by

$$
i_{b}(x, t)=i_{0}+i_{\mathrm{ac}} \sin (\omega t),
$$

where $i_{\text {ac }}$ and $\omega$ are amplitude and frequency (normalised to $\omega_{\mathrm{J}}$ ) of the dimensionless driving current. The term $i_{0}$ is a dimensionless current that, in the phase string picture, represents the initial slope of the potential profile. On increasing the slope of the washboard the height of the right potential barrier reduces. Specifically, the expression for the right potential barrier height $\Delta U$ is

$$
\Delta U=2\left[\sqrt{1-i_{b}^{2}}-i_{b} \cos ^{-1}\left(i_{b}\right)\right]
$$

The unperturbed SG equation, in the absence of damping, bias, and noise, is given by

$$
\varphi_{x x}(x, t)-\varphi_{t t}(x, t)=\sin (\varphi(x, t)) .
$$

This equation admits solutions in the travelling wave form $f=\varphi(x-u t)$ [78]

$$
\varphi(x-u t)=4 \arctan \left\{\exp \left[ \pm \frac{(x-u t)}{\sqrt{1-u^{2}}}\right]\right\}
$$


where $u$ is the propagation velocity normalised to the Swihart velocity $\bar{c}$. The Swihart velocity is the characteristic propagation speed of the electromagnetic waves along the junction and is usually more than one order of magnitude smaller than the velocity of light in vacuum. Equation (11) represents a single kink, or soliton, that is a $2 \pi$ variation in the phase values. A soliton can be depicted as a string located in two neighbouring minima crossing the intermediate maximum once (see the red solid line in panel (a) of figure 1).

The signs + and - in equation (11) indicate a $2 \pi$-kink (soliton) and a $2 \pi$-antikink (antisoliton), respectively. In this framework, according to the equation [78]

$$
\varphi_{x}=\frac{2 e}{\hbar c} d H
$$

$\varphi$ gives a normalised measure of the magnetic flux through the junction, so that equation (10) can also represent the motion of a single fluxon (or antifluxon) $\Phi_{0}=h / 2 e$. If the phase evolution shows a single $2 \pi$-kink, a single fluxon will propagate along the junction.

The normalised thermal current $i_{\mathrm{T}}(x, t)$ is characterised by the well-known statistical properties of a Gaussian random process

$$
\left\langle i_{\mathrm{T}}(x, t)\right\rangle=0, \quad\left\langle i_{\mathrm{T}}(x, t) i_{\mathrm{T}}\left(x^{\prime}, t^{\prime}\right)\right\rangle=2 \gamma_{\mathrm{T}} \delta\left(x-x^{\prime}\right) \delta\left(t-t^{\prime}\right),
$$

where $\delta$ is the Dirac delta function and

$$
\gamma_{\mathrm{T}}=\frac{k T}{R_{N}} \frac{\omega_{c}}{I_{c}^{2}}=\frac{2 e}{\hbar} \frac{k T}{I_{\mathrm{c}}}=\frac{k T}{E_{\mathrm{J}}} .
$$

It is worth noting that the noise intensity $\gamma_{\mathrm{T}}$ can also be expressed as the ratio between the thermal energy and the Josephson coupling energy $E_{\mathrm{J}}$ (see equation (15)). This equation can be rewritten as

$$
\gamma_{\mathrm{T}}=\frac{I_{\mathrm{Th}}}{I_{\mathrm{c}}} \quad \text { where } \quad I_{\mathrm{Th}}=\frac{2 e}{\hbar} k T .
$$

Here, $I_{\mathrm{Th}}$ is the equivalent thermal noise current. Inserting the numerical values, we see that $I_{\mathrm{Th}} \simeq 0.15 \mu \mathrm{A}$ at liquid helium temperature $(T=4.2 \mathrm{~K})$.

To analyse in more detail the effect of the non-Gaussian noise on the phase dynamics, we will fix the white thermal noise current at a very low intensity. Specifically, the non-Gaussian noise current $i_{\mathrm{nG}}(x, t)$ is described by $\alpha$-stable Lévy distributions.

\section{The Lévy statistics}

Here we briefly review the concept of $\alpha$-stable Lévy distributions [80-86]. A random non-degenerate variable $X$ is stable if

$$
\forall n \in \mathbb{N}, \exists\left(a_{n}, b_{n}\right) \in \mathbb{R}^{+} \times \mathbb{R}: \quad X+b_{n}=a_{n} \sum_{j=1}^{n} X_{j},
$$


where the $X_{j}$ terms are independent copies of $X$. Moreover, $X$ is strictly stable if and only if $b_{n}=0 \forall n$. The well-known Gaussian distribution stays in this class. This definition does not provide a parametric handling form of the stable distributions. The characteristic function, however, allows us to deal with them. The general definition of a characteristic function for a random variable $X$ with an associated distribution function $F(x)$ is

$$
\phi(u)=\left\langle\mathrm{e}^{\mathrm{i} u X}\right\rangle=\int_{-\infty}^{+\infty} \mathrm{e}^{\mathrm{i} u X} \mathrm{~d} F(x) .
$$

Following this statement, a random variable $X$ is said to be stable if and only if

$$
\exists(\alpha, \sigma, \beta, \mu) \in(0,2] \times \mathbb{R}^{+} \times[-1,1] \times \mathbb{R}: \quad X \stackrel{d}{=} \sigma Z+\mu,
$$

where $Z$ is a random variable with the characteristic function

$$
\phi(u)=\left\{\begin{array}{l}
\exp \left\{-|u|^{\alpha}\left[1-\mathrm{i} \beta \tan \frac{\pi \alpha}{2}(\operatorname{sign} u)\right]\right\} \quad \alpha \neq 1 \\
\exp \left\{-|u|\left[1+\mathrm{i} \beta \frac{2}{\pi}(\operatorname{sign} u) \log |u|\right]\right\} \alpha=1
\end{array}\right.
$$

in which

$$
\operatorname{sign} u=\left\{\begin{array}{rl} 
\pm 1 & u \gtrless 0 \\
0 & u=0
\end{array}\right.
$$

represents the sign function. These distributions are symmetric around zero when $\beta=0$ and $\mu=0$. In equation (19) for the $\alpha=1$ case, $0 \cdot \log 0$ is always interpreted as $\lim _{x \rightarrow 0} x \log x=0$, giving rise to $\phi(0)=1$.

Definition (18) of $X$ requires four parameters: a stability index (or characteristic exponent) $\alpha \in(0,2]$, an asymmetry parameter $\beta \in[-1,1]$, a scale parameter $\sigma>0$ and a location parameter $\mu$. The names of these parameters indicate their physical meaning. In most of the recent literature, the notation $S_{\alpha}(\sigma, \beta, \mu)$ is used for the class of stable distributions. The stable distributions obtained setting $\sigma=1$ and $\mu=0$ are called standard. The case $\beta=0$ gives a symmetric distribution, while $\alpha$ determines how the tails of the distribution go to zero. The stability index yields the asymptotic long-tail power law for the $x$-distribution, which for $\alpha<2$ is of the $|x|^{-(1+\alpha)}$ type, while $\alpha=2$ and $\beta=0$ gives a Gaussian distribution.

Figure 2(a) shows the bell-shaped probability density functions for the symmetric stable distributions $S_{\alpha}(1,0,0)$ with $\alpha \in(0,2]$. As $\alpha$ decreases, the peaks get higher, the regions around the peak become narrower (the so-called limited space displacement [39, $41])$, and the tails get heavier.

Figure 2(b) shows the probability density functions for the stable distributions $S_{0.5}(1, \beta, 0)$ for $\beta \geqslant 0$. These skewed distributions are characterised by the right tails being heavier than the left ones. When $\beta=1$, we say that the distribution is totally skewed to the right. The behaviour of the $\beta<0$ cases are reflections of the $\beta>0$ ones, with the left tail being heavier. When $\beta=-1$ the distribution is totally skewed to the left. 
Effects of Lévy noise on the dynamics of SG solitons in long JJs
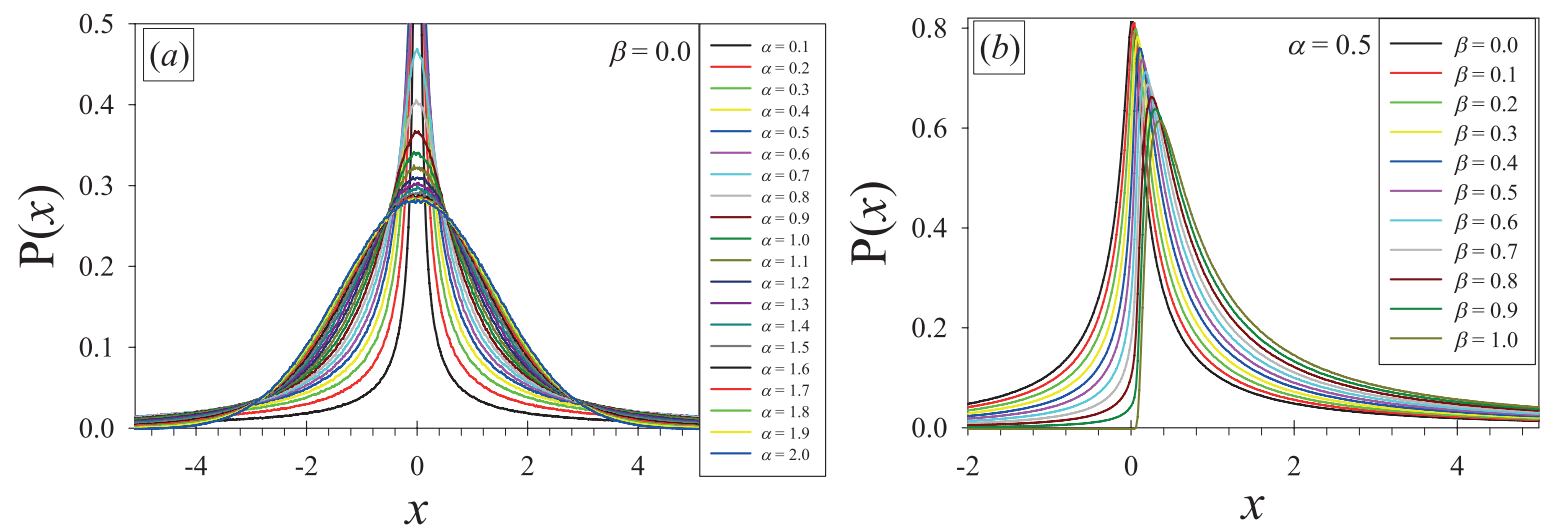

Figure 2. (a) Symmetric stable densities of $S_{\alpha}(1,0,0)$ and $\alpha \in(0,2]$. (b) Skewed stable densities of $S_{0.5}(1, \beta \geqslant 0,0)$ and $\beta \in[0,1]$.

We note that in equation (18), $\tan \left(\frac{\pi \alpha}{2}\right)=0$ for $\alpha=2$, so that the characteristic function is real and the distribution is always symmetric, apart from the value of $\beta$. As $\alpha$ decreases the effect of $\beta$ becomes more pronounced, and the left tail gets lighter and lighter for $\beta \rightarrow 1$. All stable distributions are unimodal, but there is no known formula for the location of the mode.

The heavy tails cause the occurrence of events with large values of $x$, whose probability densities are not negligible. The use of heavy-tailed statistics allows the consideration of rare events corresponding to large values of $x$ because of the fat tails of these distributions. These events correspond to the Lévy flights previously discussed. The algorithm used in this work to simulate Lévy noise sources is that proposed by Weron [87] for the implementation of the Chambers method [88].

\section{Computational details}

We study the phase dynamics of a long JJ within the SG overdamped regime, setting $\beta_{c}=0.01$. The time and spatial steps are $\Delta t=\Delta x=0.05$. Due to the stochastic nature of the dynamics of the system, we calculate the mean values of the variables analysed performing a suitable number $N=\left(5 \times 10^{3}-10^{5}\right)$ of numerical realisations (experiments). Throughout this paper we use the words string to refer to the entire junction and cell to indicate each of the elements with dimension $\Delta x$ forming the junction. The string at rest within the washboard potential valley labelled with $n=0$ (see equation (7) and panel (b) of figure 1) is chosen as an initial condition for solving equation (2), i.e. $\varphi_{0}=\arcsin \left(i_{b}(0)\right)=\arcsin \left(i_{0}\right)$. We calculate the mean switching time (MST) towards the resistive state, starting from the metastable state (the bottom of a potential minimum) corresponding to the superconducting regime. The MST $\tau$ is a nonlinear relaxation time (NLRT) [89] and represents the mean value of the permanence times of the phase $\varphi$ within the first valley, that is $\varphi \in\left[\varphi_{\text {Max }}^{\mathrm{L}}, \varphi_{\text {Max }}^{\mathrm{R}}\right]$. The thresholds $\varphi_{\text {Max }}^{\mathrm{L}}$ and $\varphi_{\text {Max }}^{\mathrm{R}}$ are, respectively, the positions of the left and right maxima, which surround the minimum chosen as an initial condition (see the dotted lines in panel (b) of figure 1). 
No absorbing barriers are set, so that during the entire observation time, $t_{\max }$, all the temporary trapping events are taken into account to calculate $\tau$. The probability $P_{i j}$ that $\varphi \in\left[\varphi_{\text {Max }}^{\mathrm{L}}, \varphi_{\text {Max }}^{\mathrm{R}}\right]$, in the $i$ th realisation for the $j$ th cell, is

$$
P_{i j}(t)=\left\{\begin{array}{l}
1 \Longleftrightarrow \varphi \in\left[\varphi_{\text {Max }}^{\mathrm{L}}, \varphi_{\text {Max }}^{\mathrm{R}}\right] \\
0 \Longleftrightarrow \varphi \notin\left[\varphi_{\text {Max }}^{\mathrm{L}}, \varphi_{\text {Max }}^{\mathrm{R}}\right] .
\end{array}\right.
$$

Summing $P_{i j}(t)$ over the total number $N_{c}=L / \Delta x$ ( $L$ is the junction length) of cells and over the number $N$ of realisations, the average probability that the entire string is in the superconducting state at time $t$ can be computed as

$$
\bar{P}(t)=\frac{1}{N N_{c}} \sum_{i=1}^{N} \sum_{j=1}^{N_{c}} P_{i j}(t) .
$$

The MST $\tau$ is therefore calculated as

$$
\tau=\int_{0}^{t_{\max }} \bar{P}(t) \mathrm{d} t
$$

The whole procedure is repeated varying the value of $\alpha$ and $\beta$, and obtaining the behaviours of the MST $\tau$ in the presence of different sources of Lévy noise.

\section{Results}

In this section, we investigate the dependence of the $\operatorname{MST} \tau$, the mean potential difference (MPD) $\zeta$, and the mean soliton densities, $n$ and $n_{\text {tot }}$, on the junction length $L$, the driving frequency $\omega$, and the noise amplitude $\gamma$ as the Lévy parameters $\alpha$ and $\beta$ are varied.

The mean soliton density $n$ is obtained considering only solitons partially lying in the potential well chosen as the initial condition (see figure 1(a)). Instead, the total mean soliton density $n_{\text {tot }}$ is obtained considering all solitons formed along the junction. These densities are calculated taking into account both kinks and antikinks.

The noise intensity $\gamma$ refers to the non-Gaussian component of the noisy current, while hereinafter the intensity of the thermal contribution is set to $\gamma_{\mathrm{T}}=10^{-4}$.

The amplitude of the oscillating component of the driving current is set to $i_{\mathrm{ac}}=0.7$ to allow, within a driving period, values of $i_{b}(t)$ greater than 1 , corresponding to the absence of metastable states.

\subsection{Results as a function of $L$}

In this section we study the behaviour of a long junction varying its length $L$. The analysis can be split in two parts: (i) a comparison between the MST $\tau$ and the mean soliton density $n$; (ii) the study of the mean potential difference (MPD) $\zeta$ across the junction related to the total mean soliton density $n_{\text {tot. }}$ The MPD is in units of $\Phi_{0}$ and is normalised to the junction length $L$. 

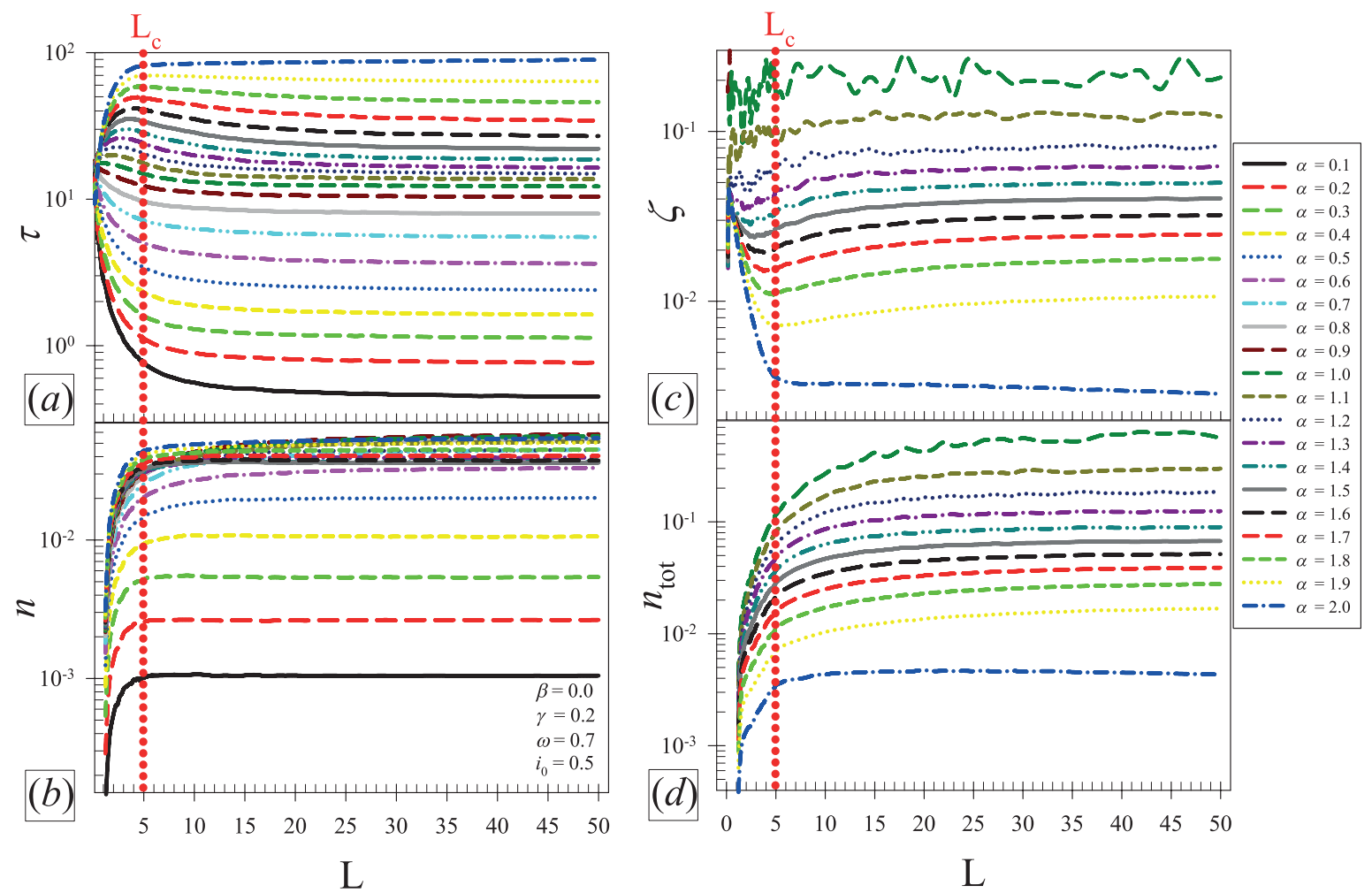

Figure 3. MST $\tau$ (panel (a)) and mean soliton density $n$ (panel (b)) as a function

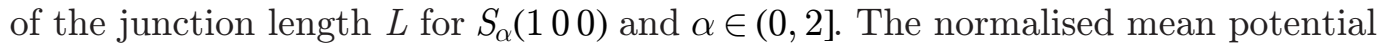
difference (MPD) $\zeta$ (see equation (27)) (panel (c)) and the mean total soliton density $n_{\text {tot }}$ (panel (d)), as a function of the junction length $L$ for $S_{\alpha}(1,0,0)$ and $\alpha \in[1,2]$. The values of the other parameters, $\gamma=0.2, \omega=0.7$ and $i_{0}=0.5$, are shown in panel (b) and refer to all the panels. The dotted vertical lines mark the nucleation length $L_{c} \simeq 5$. The legend refers to all the panels.

The MSTs as a function of the length $L$, varying the stability index $\alpha \in(0,2]$, are shown in panel (a) of figure 3 . The values of the other variables, used to compute all the curves of figure 3 , are $\beta=0, \gamma=0.2, \omega=0.7$ and $i_{0}=0.5$. The values of the MST decrease by reducing $\alpha$, because the effect of the Lévy flights become more relevant reducing $\alpha$. All the curves of panel (a) of figure 3 are characterised by the presence of two different 'dynamical regimes', in correspondence with the values of lengths below and above a threshold, namely the critical, or nucleation, length $L_{c}$ (the dotted red lines in figure 3). An initial monotonic behaviour is followed by a constant plateau in the MST curves. Specifically, for $i_{0}=0.5, L_{c} \sim 5[35,41]$.

For values of $L<L_{c}$, the soliton formation is hampered by the strength of interaction between neighbouring cells. Strings shorter than the nucleation length are indeed too small/too stiff to form ripples ample enough to overcome the potential barriers. As a consequence, below this threshold, the cells forming a string can only cross a barrier altogether, thereby reducing the chances of soliton formation. For $L<L_{c}$, curves in figure 1(a) show a monotonic behaviour of the MSTs as a function of $L$. However, this dependence changes qualitatively with $\alpha$. The MSTs either increase or decrease depending on whether $\alpha$ is below or above 1 . When $\alpha \in(1,2]$, moving rigidly a string 
across a barrier requires a bigger effort as its length increases. Hence, the MST grows with $L$, up to its maximum in $L \simeq L_{c}$. For $\alpha \in(0,1]$ a higher number of cells implies a higher probability of generating Lévy flights strong enough to push the string out to the metastable state. Consequently, the MSTs tend to decrease as $L$ increases.

Conversely, long strings move from a potential well by the formation of kinks, antikinks and/or kink-antikink pairs. For $L>L_{c}$ a saturation effect is evident [35, 41, 75, $90,91]$. The MST reaches an almost constant value, indicating that the dynamics of the switching events is independent of the JJ length and these events are guided by the solitons. To explain this behaviour, Valenti et al [41] proposed a subdomain structure of the string, in which each subdomain is composed of a number of cells of total size approximately equal to the critical length [75]. The entire string can be thought as the sum of these subdomains and the overall escape event results in being the superimposition of the escape events of each single subdomain. Consequently, increasing the junction length, the total MST is constant because it is equal to the time evolution of the individual subdomain. This means that the number of generated solitons grows linearly with the number of subdomains, that is, with the length of the junction. Inspired by this picture, we calculate the mean soliton density $n$. First, the soliton density is calculated considering, in each experiment, all the kinks and anti-kinks partially lying in the initial metastable state, which is the same well used to calculate the MSTs. Then, the mean soliton density $n$ is obtained by averaging over the total number of experiments $N$. According to [41], we observe a saturation effect in the behaviour of $n$ as a function of $L$. These curves are shown in panel (b) of figure 3 varying $\alpha \in(0,2]$. For $L<L_{c}, n$ rapidly rises to reach a plateau for $L>L_{c}$. The saturation in the MSTs can be therefore read through the constant value assumed by the mean soliton density $n$ for a long JJ.

Depending on the junction parameters, the experimentalists can choose what to measure, either the voltage or the MST. For example, measurements of the mean 'noiseinduced' voltage may be performed easily for strong to moderate damping $\left(\beta_{c}<1\right)$. In fact, on increasing $\beta_{c}$ (underdamped regime) the maximal value of current $I_{\max }<I_{\mathrm{c}}$, above which the switching to the running state occurs, decreases, and the measured voltages also become smaller (see figure 8 of [35]). Moreover, for large $\beta_{c}$ the measurements of MSTs to the resistive running state may be performed more easily as the time between sequential single flux quantum pulses.

According to the a.c. Josephson relation [92, 93], when the string rolls down along the potential, a non-zero mean voltage across the junction appears.

$$
\frac{1}{2 \pi} \frac{\mathrm{d} \varphi(x, t)}{\mathrm{d} t}=\frac{V(x, t)}{\Phi_{0}}
$$

The potential difference across the JJ (normalised to $\Phi_{0}$ ), in the $i$ th realisation at the time $t$, is

$$
\frac{V_{i}(t)}{\Phi_{0}}=\int_{0}^{\mathrm{L}} \frac{V_{i}(x, t)}{\Phi_{0}} \mathrm{~d} x=\frac{1}{2 \pi} \int_{0}^{\mathrm{L}} \frac{\mathrm{d} \varphi(x, t)}{\mathrm{d} t} \mathrm{~d} x .
$$

Therefore, the mean voltage for the $i$ th realisation is

$$
V_{i}=\frac{\left\langle V_{i}\right\rangle}{\Phi_{0}}=\frac{1}{t_{\max }} \int_{0}^{t_{\max }} \frac{V_{i}(t)}{\Phi_{0}} \mathrm{~d} t .
$$


Averaging over the total number of experiments $N$ and dividing for the JJ length $L$, we obtain the MPD $\zeta$

$$
\zeta=\frac{\bar{V}}{L}=\frac{1}{L}\left(\frac{1}{N} \sum_{i=1}^{N} V_{i}\right) .
$$

The curves of the MPD $\zeta$ as a function of $L$, varying $\alpha \in[1,2]$, are presented in panel (c) of figure 3. The curves for $\alpha<1$ strongly fluctuate and are not included here. The results in figure 3(c) highlight an 'inverse' behaviour of the MPDs in comparison with the MSTs [35]. On decreasing $\alpha$, the time derivative $\varphi_{t}$ grows because of the Lévy flights and the MPD values increase (see equation (24)). Moreover, every MPD curve decreases for small lengths $\left(L \lesssim L_{c}\right)$ up to a minimum, above which it tends to a roughly constant value. In fact, for $L \lesssim L_{c}$ as the length of the junction increases, the stiffness of the string grows and then the time derivative $\varphi_{t}$ reduces, giving rise to a decrease in the MPD (see equation (24)).

The constant value of $\zeta$ for $L>L_{c}$ is related to the saturation effect in the values of the mean soliton density. This is calculated by ensemble average of the number of kinks and anti-kinks formed along the string, without focusing only on the initial metastable state. We call this quantity the total mean soliton density $n_{\text {tot }}$. The values of $n_{\text {tot }}$ as a function of $L$, varying $\alpha \in[1,2]$, are shown in panel (d) of figure 3 . We observe that $n_{\text {tot }}$ rapidly grows for $L<L_{c}$, but above this threshold length, i.e. $L>L_{c}$, the $n_{\text {tot }}$ value tends to a constant value. Comparing the curves of panels (c) and (d) for $L>L_{c}$, we observe that $n_{\text {tot }}$ closely maps the behaviour of the MPD $\zeta$. We note that the saturation effect in both $n$ and $n_{\text {tot }}$ is proportional to the number of subdomains giving rise to solitons along the string.

The curves in panel (b) of figure 3 hide a non-monotonic behaviour as a function of $\alpha$. The values of $n$ versus $\alpha$ for $L=20, \omega=0.7$ and $i_{0}=0.5$ are shown in panel (a) of figure 4 , for three different noise intensities, namely $\gamma=0.08,0.2,1.0$. The data for $\gamma=0.2$ (red triangles) are selected from figure $3(\mathrm{~b})$ for $L=20$, and have a maximum for $\alpha=0.9$. The position of this maximum changes with the noise amplitude. Specifically, it is centered in $\alpha=0.8$ for $\gamma=0.08$, and in $\alpha=1.2$ for $\gamma=1.0$. The mean soliton density $n$ takes into account the solitons formed with respect to the first washboard valley. We can rightly assume that these solitons are most likely generated by direct flights from the first to the second washboard valley. We define the probability of 'jumping' from the initial minimum to the second one as

$$
P^{\alpha, \gamma}=\operatorname{Prob}\left\{\Delta_{1}<D^{\alpha, \gamma} x<\Delta_{2}\right\}=\operatorname{Prob}\left\{\Lambda_{1}^{\alpha, \gamma}<x<\Lambda_{2}^{\alpha, \gamma}\right\} .
$$

Here $\Delta_{1}$ and $\Delta_{2}$ are, respectively, the distances along the potential profile between the initial minimum and the two successive maxima on the right (see panel (b) of figure 1), $\Lambda_{1}^{\alpha, \gamma}=\Delta_{1} / D^{\alpha, \gamma}, \Lambda_{2}^{\alpha, \gamma}=\Delta_{2} / D^{\alpha, \gamma}$ and $D^{\alpha, \gamma}=(2 \gamma)^{1 / \alpha}$ is the Lévy noise amplitude [39]. In panels (b) and (c) of figure 3, the values of $P^{\alpha, \gamma}$ as a function of $\alpha$ for $\gamma=0.08,0.2$ (panel (b)) and $\gamma=1.0$ (panel (c)) are shown. The probabilities $P^{\alpha, \gamma}$ show non-monotonic behaviours as a function of $\alpha$, with maxima in $\alpha=0.6$ for $\gamma=0.08$, $\alpha=0.8$ for $\gamma=0.2$, and $\alpha=1.3$ for $\gamma=1.0$. The presence of these maxima in the jump probability $P^{\alpha, \gamma}$, which is a proxy of the probability to generate solitons, accounts for the non-monotonic behaviours of $n$ as a function of $\alpha$, as shown in figure 4(a). 

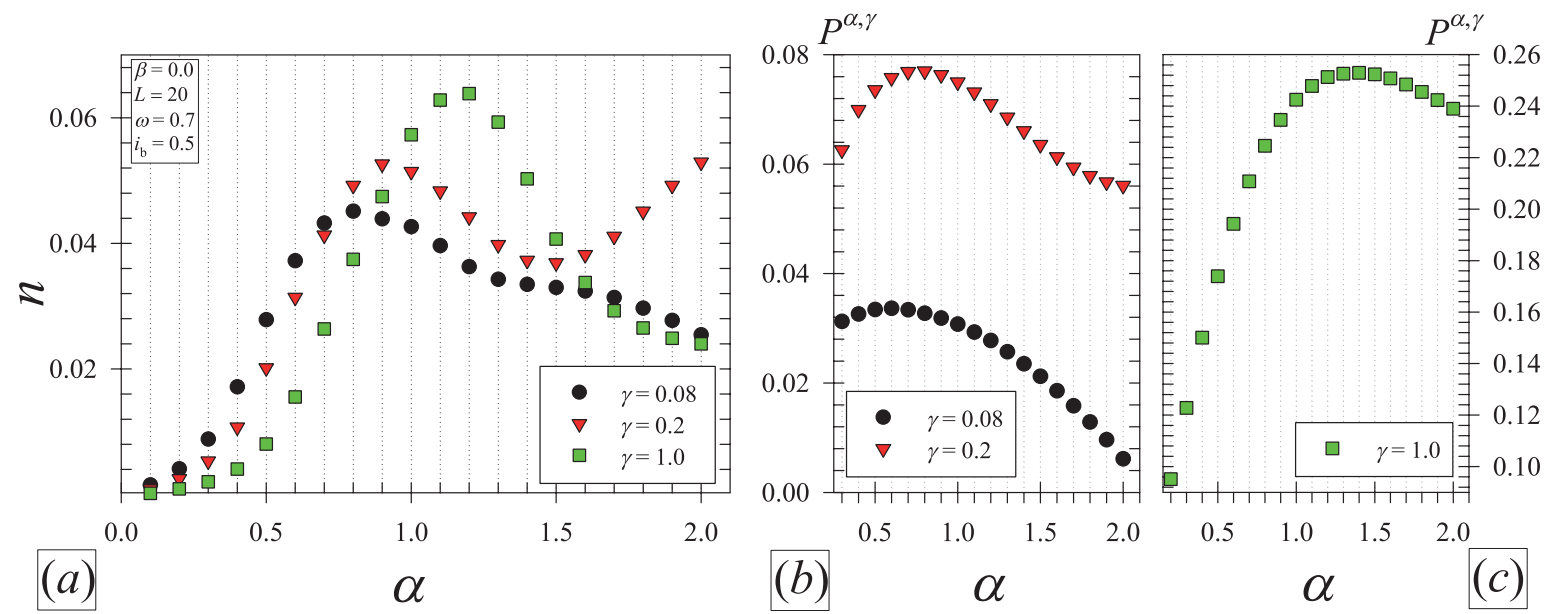

Figure 4. (a) Mean soliton density $n$ as a function of the stability index $\alpha$ for $S_{\alpha}(1,0,0)$ and $\alpha \in(0,2]$ varying the noise intensity $\gamma=0.08,0.2,1.0$. The values of the other parameters are $L=20, \omega=0.7$ and $i_{0}=0.5(\mathrm{~b}, \mathrm{c})$. Probability $P^{\alpha, \gamma}$ of obtaining values of the random variable $x$ within the range $\left(\Lambda_{1}^{\alpha, \gamma}, \Lambda_{2}^{\alpha, \gamma}\right)$ (see equation (28)) as a function of $\alpha$, for $\gamma=0.08,0.2$ (panel (b)) and $\gamma=1.0$ (panel (c)). Here $\Lambda_{1}^{\alpha, \gamma}=\Delta_{1} / D^{\alpha, \gamma}$ and $\Lambda_{2}^{\alpha, \gamma}=\Delta_{2} / D^{\alpha, \gamma}$, where $\Delta_{1}$ and $\Delta_{2}$ are, respectively, the distances between the initial minimum and the two successive maxima on the right (see panel (b) of figure 1) and $D^{\alpha, \gamma}=(2 \gamma)^{1 / \alpha}$ is the Lévy noise amplitude.

\subsection{Results as a function of $\omega$}

In this section we analyse the behaviour of the MSTs as a function of the driving frequency $\omega$. The results for different values of $\alpha \in(0,2]$ are shown in panel (a) of figure 5 setting $\beta=0.0, L=10, \gamma=0.1$ and $i_{0}=0.9$. The junction is long enough for solitons to be observed.

All curves of figure 5(a) clearly show the presence of resonant activation (RA) [41, 89, 94-102], specifically stochastic resonance activation, a noise-induced phenomenon, whose signature is the appearance of a minimum in the curve of the MST versus $\omega$. When the noise intensities are greater than $\overline{\Delta U}_{i_{0}}$ (see equation (9)), that is, the time average over a driving period of the potential barrier height, the minimum tends to vanish reducing $\alpha$ (see figure 6(c) of [41]). Accordingly, using $i_{0}=0.9$ the time average of the potential barrier height is $\overline{\Delta U}_{i_{0}=0.9} \simeq 0.4$, and we set $\gamma=0.1$.

The RA phenomenon is robust enough to be observed also in the presence of Lévy noise sources [39-42]. The particle escape from a potential well is driven when the potential barrier oscillates on a time-scale characteristic of the particle escape itself. Since the resonant frequency is close to the inverse of the average escape time at the minimum, which is the mean escape time over the potential barrier in the lowest configuration, stochastic resonant activation occurs [14, 38, 41, 103]. This phenomenon is different from the dynamic resonant activation, which is observed when the driving frequency matches the natural frequency of the system, i.e. the plasma frequency [38, 104-106].

Considering the curves in figure 5(a), we note that the RA minima shift towards higher frequencies, reducing $\alpha$. Moreover, all these curves are characterised by a 


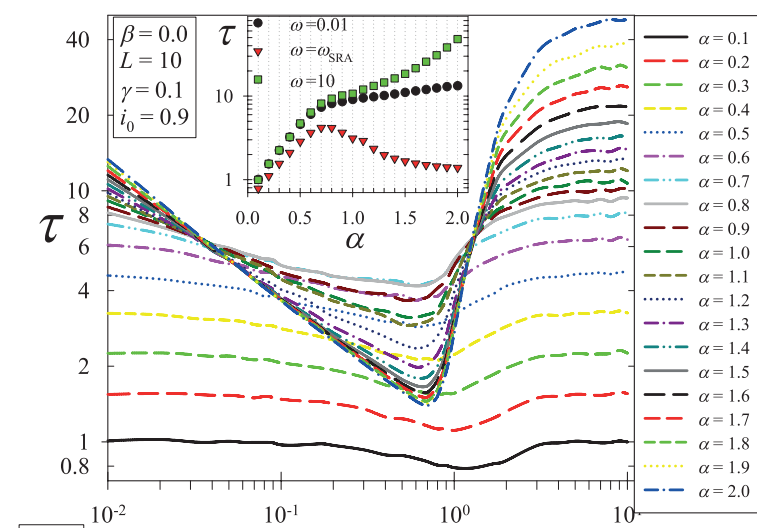

(a)

()

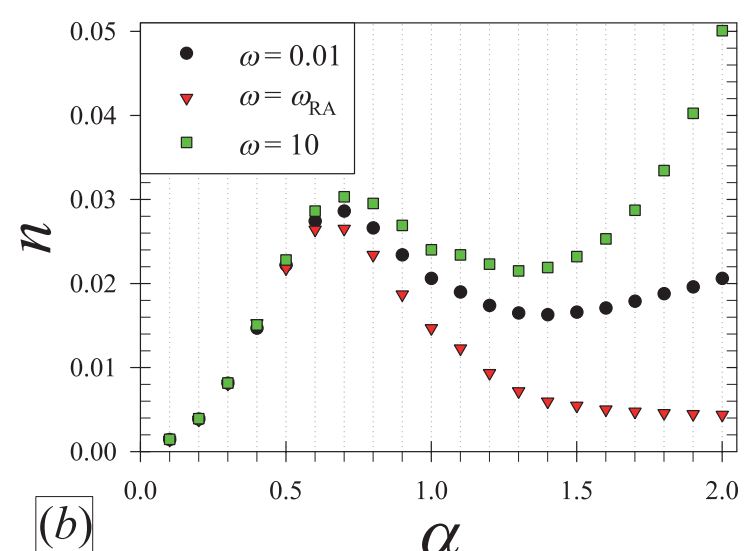

Figure 5. (a) MST $\tau$ as a function of the driving frequency $\omega$ for $S_{\alpha}(1,0,0)$ and $\alpha \in(0,2]$. The inset shows the values of the MSTs as a function of $\alpha$ for a low frequency $\omega=0.01$, for the frequencies of the RA minima $\omega=\omega_{\mathrm{RA}}$, and for a high frequency $\omega=10$. (b) Mean soliton density $n$ as a function of $\alpha$, for $S_{\alpha}(100)$ and $\alpha \in(0,2]$ and for $\omega=0.01, \omega_{\mathrm{RA}}, 10$. The values of the other parameters, $L=10$, $\gamma=0.1$ and $i_{0}=0.9$, are shown in panel (a) and refer to both panels.

non-monotonic behaviour as a function of $\alpha$, for frequencies within the range $(0.04$, 1.3). This behaviour is highlighted in the inset of figure 5(a), which shows the values of the MSTs as a function of $\alpha$ for low and high frequencies, $\omega=0.01$ and $\omega=10$, and for the frequency in the RA minima $\omega=\omega_{\mathrm{RA}}$. The curve for $\omega=\omega_{\mathrm{RA}}$ has a maximum for $\alpha=0.7$. A similar behaviour is observed looking at the mean soliton densities $n$ versus $\alpha$ presented in panel (b) of figure 5. This graph shows the results for $\omega=0.01, \omega_{\mathrm{RA}}, 10$. All these curves have a maximum located in $\alpha_{\max }=0.7$. We observe that changes in the value of the driving frequency slightly affect both the position and the height of the $n$ maxima, as well as the values of $n$ for $\alpha \leqslant \alpha_{\max }$. Conversely, for $\alpha>\alpha_{\max }$, the curves in figure 5(b) do not show the same behaviour. This can be explained by observing that, for $\alpha \leqslant \alpha_{\max }$, the behaviour of the string is closely related to the heavy tail characteristics of the Lévy noise, and the density of solitons rises with $\alpha$ reaching a maximum when the probability $P^{\alpha, \gamma}$ is the highest. Further increasing $\alpha$, the effect of the Lévy jumps reduces, but solitons can be still be created as a result of escape events after oscillations of the cells within the minimum. This explains the frequency-dependence of the mean soliton density $n$ for $\alpha>\alpha_{\max }$ (see figure 5(b)). For $\omega=\omega_{\mathrm{RA}}$, the resonant escape events are so rapid that the soliton formation is hindered, and the mean soliton density tends to low values. For off-resonance frequencies, temporary trapping of the phase occurs. As a consequence, the mean soliton density $n$ increases, and this effect is greater for high frequencies.

The MSTs versus $\omega$ for different values of $\beta \in[-1,1]$ are shown in panel (a) of figure 6 setting $\alpha=0.5, L=10, \gamma=0.1$ and $i_{0}=0.9$. The RA phenomenon is still present for all values of $\beta$, but the frequency in correspondence with the minimum grows on increasing $\beta$.

We note that a general lowering of $\tau$ occurs with increasing $\beta$. This behaviour is related to the asymmetric fluctuations that these noise sources induce for $\beta \neq 0$. For $\beta<0$, the Lévy jumps push the string in the negative $\varphi$ direction, that is, in the opposite 
Effects of Lévy noise on the dynamics of SG solitons in long JJs

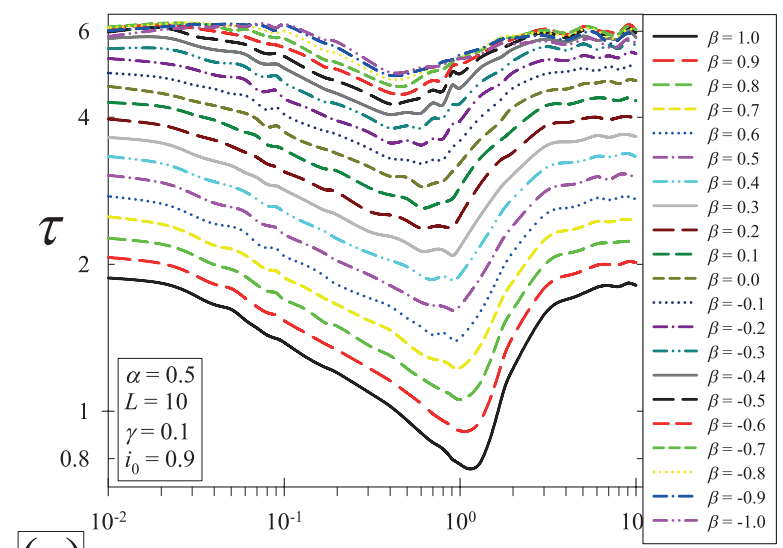

(a) $\quad \omega$

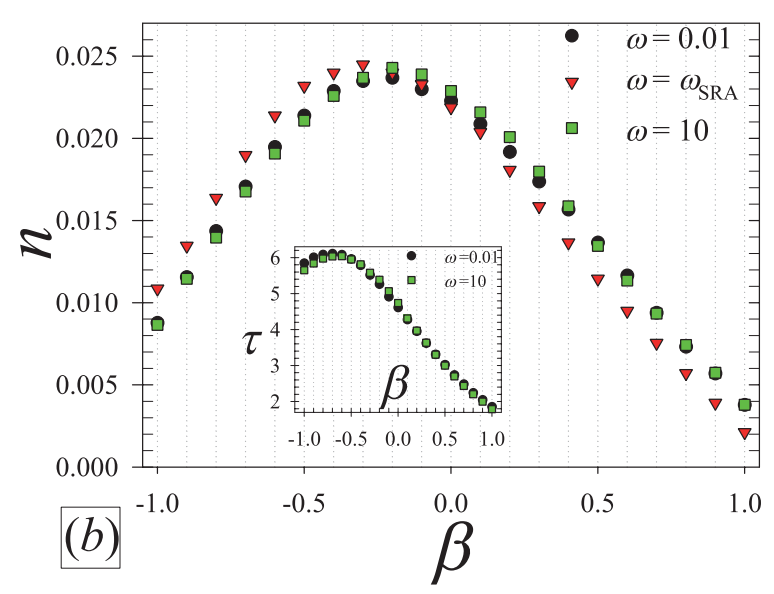

Figure 6. (a) MST $\tau$ as a function of the driving frequency $\omega$ for $S_{0.5}(1, \beta, 0)$ and $\beta \in[-1,1]$. (b) Mean soliton density $n$ as a function of $\beta$ for $S_{0.5}(1, \beta, 0)$ and $\beta \in[-1,1]$, and for $\omega=0.01, \omega_{\mathrm{RA}}, 10$. The inset shows the values of the MSTs as a function of $\beta$ for low and high frequencies, $\omega=0.01$ and $\omega=10$. The values of the other parameters, $L=10, \gamma=0.1$, and $i_{0}=0.9$ are shown in panel (a) and refer to both panels.

direction with respect to the tilting imposed by the positive bias current. Therefore, the confinement of the string within the initial metastable state is the longest for $\beta=-1$. Conversely, a positive value of $\beta$ gives fluctuations supporting the slipping of the string along the potential, resulting in very low values of $\tau$.

Panel (b) of figure 6 shows the mean soliton densities $n$ as a function of $\beta$ for $\omega=0.01, \omega_{\mathrm{RA}}, 10$. These curves are slightly affected by the value of the driving frequency, but have a maximum for $\beta_{\max }=-0.2$, which is in correspondence with a Lévy distribution only slightly skewed to the left. The nonmonotonic behaviour of $n$ versus $\beta$ is due to asymmetry properties of the Lévy noise for $\beta \neq 0$. For highly skewed distributions, that is, $\beta \gtrsim-1$ and $\beta \lesssim 1$, the escape processes occur preferentially towards the left or the right, respectively, along the washboard potential. This gives rise to low values of the mean soliton density $n$. For more symmetrical distributions, that is, $\beta \sim 0$, the escape events decrease and, as a consequence, $n$ increases. This behaviour is related to the nonmonotonic behaviour of the MST as a function of $\beta$ at low and high frequencies, as shown in the inset of figure $6(\mathrm{~b})$. At $\omega \approx \omega_{\mathrm{RA}}$, the resonant escape process drives the transient dynamics. Moreover, by varying the asymmetry parameter $\beta$ from -1 to 1 the string is pushed towards the same direction as the tilted potential, enhancing the escape process. This gives rise to a monotonic behaviour of $\tau$ as a function of $\beta$ at the resonance, see figure 6(a).

\subsection{Results as a function of $\gamma$}

In this section we analyse the MST versus the non-Gaussian noise amplitude $\gamma \in\left[10^{-4}, 5 \times 10^{2}\right]$, for different values of $\alpha$. The results for $\alpha \in(0,2], \beta=0.0, L=10$, $\omega=0.9$, and $i_{0}=0.9$ are shown in panel (a) of figure 7 .

Regardless of the value of $\alpha$, for $\gamma \rightarrow 0$, all the curves converge to the same value, i.e. the deterministic lifetime in the superconducting state, which strongly depends on the 
Effects of Lévy noise on the dynamics of SG solitons in long JJs
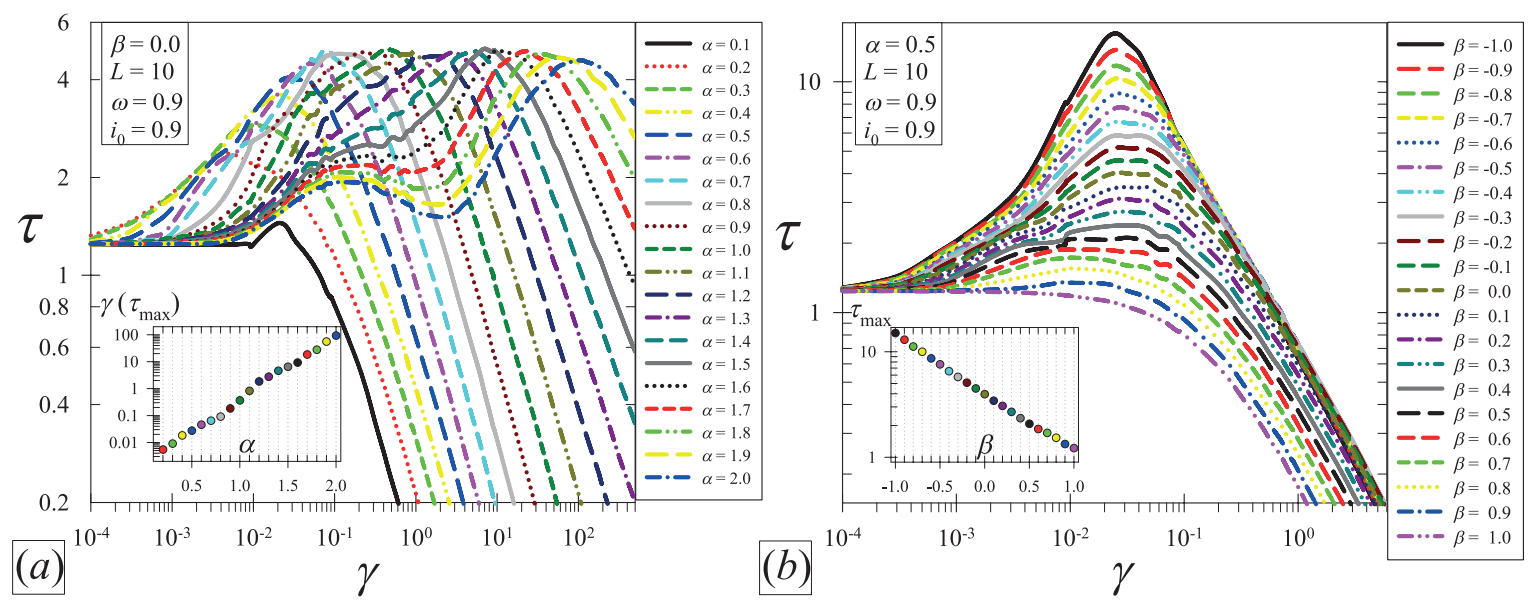

Figure 7. (a) MST $\tau$ as a function of the noise amplitude $\gamma$ for $S_{\alpha}(1,0,0)$ and $\alpha \in(0,2]$. The inset shows the position $\gamma\left(\tau_{\max }\right)$ of the NES maxima as a function of $\alpha$. (b) MST $\tau$ as a function of the noise amplitude $\gamma$ for $S_{0.5}(1, \beta, 0)$ and $\beta \in[-1,1]$. The inset shows the height $\tau_{\max }$ of the NES maxima as a function of $\beta$. The values of the other parameters are $L=10, \omega=0.9$, and $i_{0}=0.9$.

bias current. Increasing the intensity of the noise, the MST curves exhibit an effect of noise-enhanced stability (NES) [41, 89, 107-120]. This is a noise-induced phenomenon consisting of a nonmonotonic behaviour as a function of the noise intensity with the appearance of a maximum. This implies that the stability of metastable states can be enhanced and the average lifetime of the metastable state increases nonmonotonically with the noise intensity. The observed nonmonotonic resonance-like behaviour disagrees with the monotonic behaviour of Kramers theory and its extensions [121-123]. This enhancement of stability, first noted by Hirsch et al [124], has been observed in different physical and biological systems, and belongs to a highly topical interdisciplinary research field, ranging from condensed matter physics to molecular biology and to cancer growth dynamics [113, 125].

We observe that in the curve for $\alpha=2$ two maxima are present in $\gamma \simeq 0.1$ and $\gamma \simeq 100$. Reducing the value of $\alpha$ the higher maximum shifts towards smaller $\gamma$ maintaining its height, up to merge with the first maximum. In particular, the position $\gamma\left(\tau_{\max }\right)$ of the second NES peak grows exponentially towards higher noise intensities as the value of $\alpha$ increases (see the inset of figure 7(a)).

The double maxima NES for short and long JJs in the presence of a Gaussian noise source, i.e. $\alpha=2$ and $\beta=0.0$, was previously observed by Valenti et al [41]. In view of understanding the physical motivations of these NES effects, they calculated the time evolution of the probability $\bar{P}(t)$, as defined in equation (22), during the switching dynamics of the junction (see panel (b) of figure 8 in [41]). In correspondence with the first maximum, the contemporaneous presence of the oscillating potential and the noise source hinders the phase switching and therefore the passage of the junction to the resistive regime. The exit from the first well is not sharp and $\bar{P}(t)$ assumes an oscillatory behaviour, almost in resonance with the periodical motion of the washboard potential. This oscillating behaviour of $\bar{P}(t)$ tends to disappear as the noise intensity increases. In correspondence with the second NES peak for higher noise intensities no oscillations in $\bar{P}(t)$ are present. The JJ dynamics is totally driven by the noise, and the 


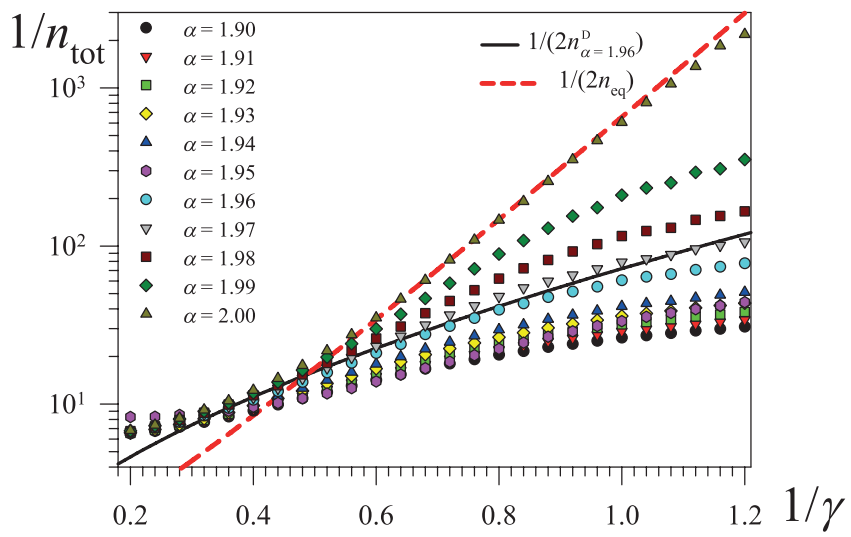

Figure 8. Inverse mean density of solitons $1 / n_{\text {tot }}$ as a function of inverse noise intensity $1 / \gamma$ in the absence of bias force $i_{b}=0$, for $S_{\alpha}(1,0,0)$ and $\alpha \in[1.9,2.0]$, and for $L=40$. The red dashed line is the inverse of the equilibrium soliton density $2 n_{\mathrm{eq}}$ calculated according to equation (29) [37, 75, 128, 129] and the black solid line is obtained from equation (29) by replacing $\gamma$ with the Lévy noise amplitude $D=(2 \gamma)^{1 / \alpha}$ with $\alpha=1.96$.

NES effect is due to the possibility that the phase string comes back into the first valley after a first escape event, as indicated by the fat tail of $\bar{P}(t)$ (see figure 8(b) of [41]).

Reducing $\alpha$, the probability of obtaining intense noise fluctuations grows, so that temporary confinements within the initial metastable state after a first escape can still occur but in correspondence with lower intensities of noise. The second NES maximum is therefore present also for $\alpha<2$, as shown in figure 7(a).

Panel (b) of figure 7 shows the MSTs as a function of $\gamma$, for different values of $\beta \in[-1,1]$, for $\alpha=0.5, L=10, \omega=0.9$ and $i_{0}=0.9$. The NES effect is still evident, although it is strongly affected by the $\beta$ value. In fact, the time of confinement of the string in the initial metastable state is longer for a Lévy noise strongly skewed in the opposite direction with respect to the positive $\varphi$ direction, that is, with respect to the tilting imposed by the positive bias current. We observe that only the height of the NES peak if affected by the $\beta$ value, whereas its position is unchanged varying $\beta$. In particular, the inset of figure 7(b) shows that the height of the NES peak exponentially decreases by increasing $\beta$.

Both panels of figure 7 show that, for high noise intensities, the MSTs have a powerlaw dependence on the noise intensity according to the expression $\tau \simeq C(\alpha) / \gamma^{\mu(\alpha)}$, where both the prefactor $\mathrm{C}$ and the exponent $\mu$ depend on the Lévy index $\alpha$ [50].

From figure 7 , we obtain $\mu(\alpha) \in(0.7,1.1)$ for $\alpha$ in $(0,2]$ and $\beta=0.0$ (see the curves in panel (a)) and $\mu(\alpha) \sim 1.1$ for $\alpha=0.5$ and $\beta \in[-1,1]$ (see the curves in panel (b)). These values of $\mu(\alpha)$ are in agreement with the exponent $\mu(\alpha) \approx 1$ for $0<\alpha<2$, calculated for barrier crossing in bistable and metastable potential profiles [126, 127].

Moreover, we calculate the total mean soliton density for the zero bias force, that is, $i_{0}=i_{\mathrm{ac}}=0$. In this condition, the solitons are exclusively generated by noise-induced fluctuations along the string. The observation time is increased to $t_{\max }=10^{3}$ and the junction length is $L=40$. The results are presented in figure 8 , where the inverse of the mean soliton density $1 / n_{\text {tot }}$ is plotted as a function of the inverse noise intensities $1 / \gamma$ 
for $\alpha \in[1.9,2.0]$ and $\beta=0.0$. We can compare the curve for $\alpha=2$ with the formula for the kink density [37, 75, 128, 129]

$$
n_{\mathrm{eq}}=\sqrt{\frac{2}{\pi} \frac{E_{k}}{\gamma}} \exp \left(-\frac{E_{k}}{\gamma}\right) .
$$

The total soliton density (kinks and antikinks) in a string is twice the kink density given in equation (29) (see $1 /\left(2 n_{\text {eq }}\right)$ curve, red dashed line, in figure 8$)$. In equation (29), the energy $E_{k}=8 E_{\mathrm{J}}$ is the rest energy of a kink (or an antikink). The total soliton density $n_{\text {tot }}$ calculated for $\alpha=2$ perfectly matches the prediction of equation (29) for $1 / \gamma \gtrsim 0.5$.

When the noise intensity is greater than the mean potential barrier height $\overline{\Delta U}_{i_{0}=0.0}=2$, i.e. $1 / \gamma \lesssim 0.5$, the curves in figure 8 overlap. For smaller noise intensities, i.e. $1 / \gamma>0.5$, the mean soliton density $n_{\text {tot }}$ definitively increases as the value of $\alpha$ slightly reduces. We observe that the behaviour of the curves for $\alpha \in[1.9,2.0)$ can be approximately estimated by replacing in equation (29) the noise intensity with the Lévy noise amplitude $D^{\alpha, \gamma}=(2 \gamma)^{1 / \alpha}$ (see the black solid line in figure 8 for $\alpha=1.96$ ).

\section{Conclusions}

We studied how both non-Gaussian random fluctuations and an oscillating driving contribute to the generation of solitons in a long Josephson junction (also called a string), which is a system governed by the sine-Gordon model. The non-Gaussian noise sources are modelled by using $\alpha$-stable Lévy statistics. In detail, we analysed the behaviour of the superconducting lifetime and the voltage drop across the junction as the values of the characteristic Lévy parameters $\alpha$ and $\beta$ change. The superconducting lifetime of the junction is calculated as the mean switching time (MST) from the initial metastable state, i.e. a minimum of the tilted washboard potential.

Studying the MST as a function of the junction length $L$, two different behaviours are observed, in correspondence with regimes of length below and above a critical value $L_{c}$. One, occurring for short junctions, characterised by the movement of the phase string as a whole. The other one, occurring for junctions whose size exceeds $L_{c}$, in which the soliton creation is allowed. We found a connection between the behaviour of the MST for $L>L_{c}$ and the mean density $n$ of the solitons partially lying in the initial metastable state. Moreover, we observed that, for a fixed length of the junction, $n$ varies nonmonotonically as a function of $\alpha$, showing a clear maximum. A similar nonmonotonic behaviour characterises the probability $P^{\alpha, \gamma}$ of a direct 'noise-induced' jump from the initial washboard minimum to the next one.

We also investigated the mean potential difference (MPD) as a function of the junction length. For $L>L_{c}$ the MPD behaves just like the mean density $n_{\text {tot }}$ of the solitons formed along the string.

Studying the behaviour of the MST as a function of the driving frequency $\omega$, we observed the presence of stochastic resonance activation, that is, a noise-induced phenomenon whose signature is the appearance of a minimum in the curve of the MST 
versus $\omega$. For frequencies near the RA minima, the behaviour of the MST as a function of $\alpha$ is characterised by the presence of a maximum. We understood this result studying the jump probability $P^{\alpha, \gamma}$, which shows the same nonmonotonic behaviour as a function of $\alpha$.

We found evidence of a nonmonotonic behaviour also by studying the MSTs as a function of the noise intensity $\gamma$, observing the phenomenon of noise-enhanced stability, whose characteristics strongly depend on the values of the Lévy parameters $\alpha$ and $\beta$.

Our findings are important to understand the role of non-Gaussian noise sources on the transient dynamics of out of equilibrium long JJ devices. This is important not only in the general context of nonequilibrium statistical mechanics, but also to improve the performance of these devices. Moreover, the statistics of the escape times from the superconductive metastable state of a JJ carries information on the non-Gaussian background noise [41].

\section{References}

[1] Helström C 1968 Statistical Theory of Detection (Oxford: Pergamon)

[2] Jaranowski P, Królak A and Schutz B F 1998 Phys. Rev. D 58063001

[3] Salter C J, Emerson D T, Steppe H and Thum C 1989 Astron. Astrophys. 225 167-78

[4] Mittleman M D 2003 Sensing with Terahertz Radiation (Berlin: Springer)

[5] Inchiosa M E and Bulsara A R 1996 Phys. Rev. E 53 R2021-4

[6] Gammaitoni L, Hänggi P, Jung P and Marchesoni F 1998 Rev. Mod. Phys. 70 223-87

[7] Galdi V, Pierro V and Pinto I M 1998 Phys. Rev. E 57 6470-9

[8] Khudchenko A V, Koshelets V P, Dmitriev P N, Ermakov A B, Yagoubov P A and Pylypenko O M 2009 Supercond. Sci. Technol. 22085012

[9] Ozyuzer L et al 2007 Science 318 1291-3

[10] Castellano M G, Leoni R, Torrioli G, Chiarello F, Cosmelli C, Costantini A, DiambriniPalazzi G, Carelli P, Cristiano R and Frunzio L 1996 J. Appl. Phys. 80 2922-8

[11] Glukhov A M, Sivakov A G and Ustinov A V 2002 Low Temp. Phys. 28 383-6

[12] Grønbech-Jensen N, Castellano M G, Chiarello F, Cirillo M, Cosmelli C, Filippenko L V, Russo R and Torrioli G 2004 Phys. Rev. Lett. 93107002

[13] Filatrella G and Pierro V 2010 Phys. Rev. E 82046712

[14] Addesso P, Filatrella G and Pierro V 2012 Phys. Rev. E 85016708

[15] Addesso P, Pierro V and Filatrella G 2013 Europhys. Lett. 10120005

[16] Pierro V and Filatrella G 2015 Physica C 517 37-40

[17] Addesso P, Pierro V and Filatrella G 2016 Commun. Nonlinear Sci. Numer. Simul. 30 15-31

[18] Grabert H 2008 Phys. Rev. B 77205315

[19] Urban D F and Grabert H 2009 Phys. Rev. B 79113102

[20] Ankerhold J 2007 Phys. Rev. Lett. 98036601

[21] Sukhorukov E V and Jordan A N 2007 Phys. Rev. Lett. 98136803

[22] Köpke M and Ankerhold J 2013 New J. Phys. 15

[23] Huard B, Pothier H, Birge N O, Esteve D, Waintal X and Ankerhold J 2007 Ann. Phys. 16 736-50

[24] Peltonen J T, Timofeev A V, Meschke M, Heikkilä T T and Pekola J P 2007 Physica E 40 111-22

[25] Montroll E W and Shlesinger M F 1984 Nonequilibrium Phenomena II: from Stochastics to Hydrodynamics (Amsterdam: North-Holland)

[26] Shlesinger M F, Zaslavsky G M and Frisch U 1995 Lévy Flights and Related Topics in Physics (Berlin: Springer)

[27] Dybiec B and Gudowska-Nowak E 2004 Phys. Rev. E 69016105

[28] Souryal M R, Larsson E G, Peric B and Vojcic B R 2008 IEEE Trans. Signal Process. 56 266-73

[29] Gordeeva A V and Pankratov A L 2006 Appl. Phys. Lett. 88022505

[30] Gordeeva A V and Pankratov A L 2008 J. Appl. Phys. 103103913

[31] Pankratov A L and Spagnolo B 2004 Phys. Rev. Lett. 93177001

[32] Augello G, Valenti D and Spagnolo B 2008 Int. J. Quantum Inf. 6 801-6 
[33] Gordeeva A V, Pankratov A L and Spagnolo B 2008 Int. J. Bifurcation Chaos 18 2825-31

[34] Augello G, Valenti D, Pankratov A L and Spagnolo B 2009 Eur. Phys. J. B 70 145-51

[35] Fedorov K G and Pankratov A 2007 Phys. Rev. B 76024504

[36] Fedorov K G, Pankratov A L and Spagnolo B 2008 Int. J. Bifurcation Chaos 18 2857-62

[37] Fedorov K G and Pankratov A L 2009 Phys. Rev. Lett. 103260601

[38] Guarcello C, Valenti D and Spagnolo B 2015 Phys. Rev. B 92174519

[39] Augello G, Valenti D and Spagnolo B 2010 Eur. Phys. J. B 78 225-34

[40] Guarcello C, Valenti D, Augello G and Spagnolo B 2013 Acta Phys. Pol. B 44 997-1005

[41] Valenti D, Guarcello C and Spagnolo B 2014 Phys. Rev. B 89214510

[42] Spagnolo B, Valenti D, Guarcello C, Carollo A, Persano Adorno D, Spezia S, Pizzolato N and Di Paola B 2015 Chaos Solitons Fractals 81 412-24

[43] Guarcello C, Valenti D, Carollo A and Spagnolo B 2015 Entropy 172862

[44] Szajnowski W J and Wynne J B 2001 IEEE Signal Process. Lett. 8 151-2

[45] Chechkin A V, Gonchar V Y, Klafter J and Metzler R 2006 Adv. Chem. Phys. B 133 439-96

[46] Metzler R and Klafter J 2000 Phys. Rep. 3391

[47] Uchaikin V V 2003 Phys.-Usp 46 821-49

[48] Dubkov A A, La Cognata A and Spagnolo B 2009 J. Stat. Mech. P01002

[49] Dubkov A A and Spagnolo B 2005 Fluct. Noise Lett. 05 L267-74

[50] Dubkov A A, Spagnolo B and Uchaikin V 2008 Int. J. Bifurcation Chaos Appl. Sci. Eng. 18 2649-72

[51] Woyczynski W A 2001 Lévy Processes: Theory and Applications (Boston, MA: Birkhauser)

[52] Dubkov A A and Spagnolo B 2007 Acta Phys. Pol. B 38 1745-58

[53] Dubkov A A and Spagnolo B 2013 Eur. Phys. J. Spec. Top. 216 31-5

[54] Sims D W 2008 Nature 451 1098-102

[55] Reynolds A 2008 Ecology $892347-51$

[56] West B J and Deering W 1994 Phys. Rep. 246 1-100

[57] Lan B L and Toda M 2013 Eur. Phys. Lett. 10218002

[58] Lisowski B, Valenti D, Spagnolo B, Bier M and Gudowska-Nowak E 2015 Phys. Rev. E 91042713

[59] Dubkov A A and Spagnolo B 2008 Eur. Phys. J. B 65 361-7

[60] La Cognata A, Valenti D, Dubkov A A and Spagnolo B 2010 Phys. Rev. E 82011121

[61] La Cognata A, Valenti D, Spagnolo B and Dubkov A A 2010 Eur. Phys. J. B 77 273-9

[62] Ditlevsen P D 1999 Geophys. Res. Lett. 26 1441-4

[63] Shlesinger M F 1971 Lecture Notes in Physics vol 450 (Berlin: Springer)

[64] Mantegna R N and Stanley H E 1995 Nature 376 46-9

[65] Simmross-Wattenberg F, Asensio-Perez J, Casaseca-de-la Higuera P, Martin-Fernandez M, Dimitriadis I and Alberola-Lopez C 2011 IEEE Trans. Depend. Secure 8 494-509

[66] Brockmann D, Hufnagel L and Geisel T 2006 Nature 439 462-5

[67] Subramanian A, Sundaresan A and Varshney P 2015 IEEE Trans. Signal Process. 63 2790-803

[68] Luryi S, Semyonov O, Subashiev A and Chen Z 2012 Phys. Rev. B 86201201

[69] Semyonov O, Subashiev A V, Chen Z and Luryi S 2012 J. Lumin. 132 1935-43

[70] Briskot U, Dmitriev I A and Mirlin A D 2014 Phys. Rev. B 89075414

[71] Subashiev A V, Semyonov O, Chen Z and Luryi S 2014 Phys. Lett. A 378 266-9

[72] Vermeersch B, Mohammed A M S, Pernot G, Koh Y R and Shakouri A 2014 Phys. Rev. B 90014306

[73] Nolan J P 2015 Bibliography on stable distributions, processes and related topics http://academic2.american. edu/ jpnolan/stable/stable.html

[74] Ustinov A V 1998 Physica D 123 315-29

[75] Büttiker M and Landauer R 1981 Phys. Rev. A 23 1397-410

[76] McLaughlin D W and Scott A C 1978 Phys. Rev. A 18 1652-80

[77] Dueholm B, Joergensen E, Levring O A, Monaco R, Mygind J, Pedersen N F and Samuelsen M R 1982 IEEE Trans. Magn. 19 1196-200

[78] Barone A and Paternò G 1982 Physics and Applications of the Josephson Effect (New York: Wiley)

[79] Likharev K 1986 Dynamics of Josephson Junctions and Circuits (London: Gordon and Breach)

[80] Bertoin J 1996 Lévy Processes (Cambridge: Cambridge University Press)

[81] Sato K 1999 Lévy Processes and Infinitely Divisible Distributions (Cambridge: Cambridge University Press)

[82] Kolmogorov A N and Gnedenko B 1954 Limit Distributions for Sums of Independent Random Variables (Cambridge, MA: Addison-Wesley)

[83] De Finetti B 1975 Theory of Probability vols 1 and 2 (New York: Wiley)

[84] Khintchine A and Lévy P 1936 C. R. Acad. Sci. Paris 202374

[85] Khintchine A Y 1938 Limit Distributions for the Sum of Independent Random Variables (Moscow: ONTI)

[86] Feller W 1971 An Introduction to Probability Theory and its Applications vol 2 (New York: Wiley) 
[87] Weron R 1996 Stat. Probab. Lett. 28 165-71

[88] Chambers J M, Mallows C L and Stuck B W 1976 J. Am. Stat. Assoc. 71 340-4

[89] Dubkov A A, Agudov N V and Spagnolo B 2004 Phys. Rev. E 69061103

[90] Castellano M G, Torrioli G, Cosmelli C, Costantini A, Chiarello F, Carelli P, Rotoli G, Cirillo M and Kautz R L 1996 Phys. Rev. B 54 15417-28

[91] Simanjuntak H P and Gunther L 1997 J. Phys.: Condens. Matter 92075

[92] Josephson B D 1962 Phys. Lett. 1 251-3

[93] Josephson B D 1974 Rev. Mod. Phys. 46 251-4

[94] Doering C R and Gadoua J C 1992 Phys. Rev. Lett. 69 2318-21

[95] Mantegna R N and Spagnolo B 2000 Phys. Rev. Lett. 84 3025-8

[96] Mantegna R N and Spagnolo B 1998 J. Phys. IV (France) 8 Pr6-247-Pr6-251

[97] Pechukas P and Hänggi P 1994 Phys. Rev. Lett. 73 2772-5

[98] Marchi M, Marchesoni F, Gammaitoni L, Menichella-Saetta E and Santucci S 1996 Phys. Rev. E $543479-87$

[99] Dybiec B and Gudowska-Nowak E 2009 J. Stat. Mech.: Theor. Exp. P05004

[100] Miyamoto S, Nishiguchi K, Ono Y, Itoh K M and Fujiwara A 2010 Phys. Rev. B 82033303

[101] Hasegawa Y and Arita M 2011 Phys. Lett. A 375 3450-8

[102] Fiasconaro A and Spagnolo B 2011 Phys. Rev. E 83041122

[103] Pan C, Tan X, Yu Y, Sun G, Kang L, Xu W, Chen J and Wu P 2009 Phys. Rev. E 79 R030104

[104] Devoret M H, Martinis J M, Esteve D and Clarke J 1984 Phys. Rev. Lett. 53 1260-3

[105] Devoret M H, Martinis J M and Clarke J 1985 Phys. Rev. Lett. 55 1908-11

[106] Martinis J M, Devoret M H and Clarke J 1987 Phys. Rev. B 35 4682-98

[107] Mantegna R and Spagnolo B 1996 Phys. Rev. Lett. 76 563-6

[108] Agudov N V and Spagnolo B 2001 Phys. Rev. E 64 351021-4

[109] Spagnolo B, Agudov N V and Dubkov A A 2004 Acta Phys. Pol. B 35 1419-36

[110] D'Odorico P, Laio F and Ridolfi L 2005 Proc. Natl Acad. Sci. USA 102 10819-22

[111] Fiasconaro A, Spagnolo B and Boccaletti S 2005 Phys. Rev. E 72061110

[112] Hurtado P I, Marro J and Garrido P L 2006 Phys. Rev. E 74050101

[113] Spagnolo B, Dubkov A A, Pankratov A L, Pankratova E V, Fiasconaro A and Ochab-Marcinek A 2007 Acta Phys. Pol. B 38 1925-50

[114] Mankin R, Soika E, Sauga A and Ainsaar A 2008 Phys. Rev. E 77051113

[115] Yoshimoto M, Shirahama H and Kurosawa S 2008 J. Chem. Phys. 129

[116] Fiasconaro A and Spagnolo B 2009 Phys. Rev. E 80041110

[117] Trapanese M 2009 J. Appl. Phys. 105 07D313

[118] Fiasconaro A, Mazo J J and Spagnolo B 2010 Phys. Rev. E 82041120

[119] Li J H and Łuczka J 2010 Phys. Rev. E 82041104

[120] Smirnov A A and Pankratov A L 2010 Phys. Rev. B 82132405

[121] Kramers H A 1940 Physica 7 284-304

[122] Mel'nikov V I 1991 Phys. Rep. 2091

[123] Hänggi P, Talkner P and Borkovec M 1990 Rev. Mod. Phys. 62 251-341

[124] Hirsch J E, Huberman B A and Scalapino D J 1982 Phys. Rev. A 25 519-32

[125] Spagnolo B, Caldara P, La Cognata A, Augello G, Valenti D, Fiasconaro A, Dubkov A A and Falci G 2012 Acta Phys. Pol. B 43 1169-89

[126] Chechkin A V, Gonchar V Y, Klafter J and Metzler R 2005 Europhys. Lett. 72 348-54

[127] Chechkin A V, Sliusarenko O Y, Metzler R and Klafter J 2007 Phys. Rev. E 75041101

[128] Büttiker M and Landauer R 1979 Phys. Rev. Lett. 43 1453-6

[129] Büttiker M and Christen T 1995 Phys. Rev. Lett. 75 1895-8 